\title{
A Comparative Study of Auramine O Staining Using LED Fluorescent Microscopy with Ziehl -Neelsen Staining in the Examination of Sputum For Acid Fast Bacilli
}

\author{
Authors \\ Okonkwo, R. C', Anyabolu, A. E' ${ }^{2}$, Onwunzo M. C ${ }^{1}$, Ifeanyichukwu, M. $\mathbf{O}^{3^{*}}$, \\ Chukwuka, C. $\mathbf{P}^{1}$, Enemuo, $\mathrm{E}^{2}$, Ngwu A. $\mathbf{M}^{4}$ \\ ${ }^{1}$ Department of Medical Microbiology/Tuberculosis (DOT) Laboratory, Nnamdi Azikiwe University \\ Teaching Hospital, Nnewi \\ ${ }^{2}$ Department of Internal Medicine/Tuberculosis (DOT) Laboratory, Nnamdi Azikiwe University Teaching \\ Hospital, Nnewi \\ ${ }^{3}$ Department of Immunology, College of Health Sciences, Nnamdi Azikiwe University, Nnewi Campus \\ ${ }^{4}$ Department of Hematology and Immunology, Enugu State University of Science and Technology, Enugu \\ Corresponding Author \\ Martin Ositadinma Ifeanyichukwu \\ Nnewi, Anambra State, Nigeria, PMB 5001 \\ Email: moifeanyi@yahoo.co.uk,+23408037200407
}

\begin{abstract}
.
Background: Despite all interventions, tuberculosis (TB) remains a public health issue in Nigeria. Effective TB treatment and control demand early case detection. The ZN staining technique, which is the method of choice in the DOTS program can have problem of low sensitivity. Hence, the need for more sensitive alternative methods. Use of culture is the gold standard but it is not feasible in resource limited settings. The fluorescent Light Emitting Diode (LED) microscopy (FM) method using auramine $O$ stain offers a viable alternative.

Aims and Objectives: To study the efficacy of the fluorescent LED microscopy in the diagnosis of pulmonary tuberculosis (PTB) in comparison with the $Z N$ method and to determine which of the methods is faster in the detection of Acid Fast Bacilli (AFB).

Methodology: Early morning sputum samples were collected from 630 pulmonary tuberculosis suspects (PTB) attending the TB (DOTS) centre of Nnamdi Azikiwe University Teaching Hospital, Nnewi, Nigeria. Two smears, measuring $1 \times 2 \mathrm{~cm}$ in diameter, made from each specimen received were stained, one with Ziehl Neelsen staining method and the other with auramine $O$ fluorescent staining method.

Results: Microscopy results obtained from both methods were recorded according to the IUALTD/WHO guideline as adopted by the National Tuberculosis programme (NTP). The results showed comparable sensitivities. The positivity rate was 51/630(8.1\%) for ZN and 55/630(8.7\%) for FM. However, FM was found to be faster than ZN technique.

Conclusions: The faster reading and the ease of use of the LED fluorescent microscope together with its general acceptability actually support its use in the DOTS program, not as an alternative but as an adjuvant, especially during the introductory phase which should be monitored.
\end{abstract}

Keywords: Tuberculosis, DOTS, LED, ZN. 


\section{Introduction}

Nigeria established its National Tuberculosis and Leprosy Control Program in 1989 and adopted the Directly Observed Treatment Short course (DOTS) strategy 1994 and has successfully extended the services to all 36 states and the Federal Capital Territory (FCT) by 2004, with about 3455 health facilities providing free DOTS services by $2009^{[1]}$. Despite all these, tuberculosis still constitutes a major public health issue in Nigeria, with an estimated 320,000 prevalent cases in 2010, equivalent to $199 / 100,000$ population and death rate standing at $5 \%{ }^{[2,3]}$. Effective TB control program borders on early case detection. Of the wide spectrum of procedures available for diagnosis of $\mathrm{TB}$, the sputum smear microscopy using the hot Ziehl Neelsen $(\mathrm{ZN})$ method is recommended for use in the National Tuberculosis Program (NTP) in Nigeria ${ }^{[4]}$. The method is quite attractive for public health programs. It is fast and inexpensive, requiring simple equipment and is also specific enough that no confirmatory testing is needed ${ }^{[5]}$. It provides not only visual evidence of tuberculosis but also of the bacterial burden ${ }^{[6]}$. Its smear positivity correlates well with the severity of pulmonary $\mathrm{TB}$ and the infectiousness of the patients ${ }^{[7]}$. However, this method appears to have some shortcomings that could be unfavorable to TB control targets especially in high TB settings. It is seen as tedious and time consuming, thus limiting the number of slides that can be examined per day. It is also considered to be of low and variable sensitivity when compared to other diagnostic methods. It detects fewer than $60 \%$ of all new TB infections and as few as $20-35 \%$ of HIV/TB infections ${ }^{[8]}$. According to the International Union Against Tuberculosis and Lung Disease technical guideline, at least 5 minutes of screening of a minimum of 100 high power fields is required to correctly identify a negative smear result using conventional light microscopy ${ }^{[9]}$. In busy over burdened labs, smears may not be given this minimum time, with the result that a slide may be declared negative when actually it was scanty positive (1-9AFB/100 Fields). On other occasions, given extra time for re-examination, it has been proven that negative smears stained by $\mathrm{ZN}$ method were, in fact, positive, showing the negative impact the method could have on early case detection ${ }^{[10]}$. This situation presents a special challenge in the diagnosis of TB, especially in HIV/TB co infected persons with pauci- bacillary sputum. There is the utmost need for early and accurate detection, if set TB control targets must be achieved. Hence, the quest for alternative or complimentary diagnostic procedures with enhanced sensitivity. Use of culture is the gold standard but it is not feasible in resource limited settings ${ }^{[11]}$. Several automated molecular techniques with reduced turn over time have been developed for the diagnosis of TB but they are costly and unaffordable for routine use ${ }^{[12]}$. The fluorescent microscopy method comes in as a viable alternative staining method because of its perceived enhanced sensitivity. The contrasting color of the FM makes it easier to detect positive cases at lower magnification of $400 x$, thus allowing screening of greater smear area in each field viewed with less time consumption ${ }^{[13,14]}$. The fluorescent dye enhances contrast and amplifies the observer's ability to observe stained cells 10 folds greater than light microscopy. W H $\mathrm{O}$ in 2009 recommended that LED FM be phased in as an alternative to $\mathrm{ZN}$ microscopy in both high volume and low- income laboratories [14]. Numerous reports have confirmed the superior diagnostic performance of fluorescence microscopy compared with ZN staining method [15-17]. It was therefore the aim of this study to determine the accuracy and speed of fluorescence microscopy compared with conventional $\mathrm{ZN}$ microscopy in the diagnosis of active TB in Pulmonary Tuberculosis (PTB) suspects attending the TB Directly Observed Treatment Short course (DOTS) centre of the Nnamdi Azikiwe University Teaching Hospital (NAUTH), Nnewi, Nigeria. 


\section{Methodology}

The study comprised of 630 PTB suspects attending the TB (DOTS) centre of NAUTH, Nnewi, Nigeria. Each of the 630 patients produced one early morning sputum sample into wide mouthed, transparent, leak- proof, clean sputum cup, without eating or brushing of the mouth. This was the second of the three samples required for routine sputum smear microscopy. Two smears, each measuring $1 \times 2 \mathrm{~cm}$ in diameter, were prepared from each specimen received, one for examination using $\mathrm{ZN}$ technique and other for examination with Auramine $\mathrm{O}$ staining technique. Three (3) Medical Laboratory Scientists took turn in reading the slides prepared for either bright or fluorescence microscopy. None of the 3 scientists read the slides from the same specimen and they were blinded to the results obtained with the other technique. Results were recorded according to the IUALTDWHO guidelines as adopted by the national tuberculosis program ${ }^{[4]}$. Hundred fields of each smear were examined before declaring a slide negative or scanty positive (1-9AFB/100 fields), about 50 fields for $2+$ positive and about 20 fields for slides $3+$ positive, using oil immersion(x100) objective for $\mathrm{ZN}$ stained slides while one length of 40 fields were examined using (x40) objective of the LED microscope for the auramine-O stained slides. Two smears, each measuring $1 \times 2 \mathrm{~cm}$ in diameter, were prepared from a separate batch of fifty sputum specimens, one for examination using $\mathrm{ZN}$ technique and other for examination with Auramine $\mathrm{O}$ staining technique. The mean time required to examine 50 slides using the two techniques was also determined.

\section{Results}

Results were recorded according to the IUALTDWHO guidelines as adopted by the National Tuberculosis Program and shown in table 1 . Out of the 630 slides the smear positivity rate for $\mathrm{AFB}$ using the conventional $\mathrm{ZN}$ method was $51 / 630(8.1 \%)$ while the positivity rate using the LED auramine $\mathrm{O}$ staining method was
$55 / 630(8.9 \%)$ as shown in table 2 . The mean time required to examine 50 slides using the $\mathrm{ZN}$ techniques with bright light microscope was (140/50) 2.8 minutes while that for 50 auramine O stained slides using LED fluorescent microscope was $(75 / 50) 1.5$ minutes.

\section{Discussion}

The devastating impact of TB on vulnerable populations continues to be a major public health issue in the developing countries. Building capacity and enhancing universal access to rapid, accurate and early laboratory diagnosis are very important for effective treatment and control of TB. The WHO advocates the alternative use of the Light Emitting Diode (LED) fluorescent microscopy (FM) in place of $\mathrm{ZN}$ method so as to improve the sensitivity and result generation time for smear microscopy. This present study demonstrated that auramine $\mathrm{O}$ stained smears actually take less time to examine than the $\mathrm{ZN}$ stained smears but it did not observe any significant difference in the sensitivities of the two methods. The gained time is said to be due to the fact that fluorescent stain imparts some significant contrast to AFB making them to be seen much more quickly than the red of $\mathrm{ZN}$ stained AFB in a blue back ground. There is an increased distinction which permits the use of objectives with larger fields of view and thereby decreasing total examination time ${ }^{[13] .}$ This present study tally with those of Bonnet et al, 2011, in Kenya, Nairobi and Sekhar et al in India who reported comparable sensitivities with the two methods $[17,18]$. The present study was actually our first attempt to use LED-fluorescent method and compare it with the conventional $\mathrm{ZN}$ method. Generally, the acceptability of the LED-FM was very good. The absence of an outstanding increase in sensitivity might be explained by the great experience of the study medical laboratory scientists with $\mathrm{ZN}$ method whereas they started using FM following recent training on FM. They are quite more skilled and experienced in the use of $\mathrm{ZN}$ method. Nonetheless the faster reading of 
LED-FM smears combined with the very good acceptability and ease of use would support its introduction in DOTS laboratories, starting first with those with high workload, in view of the initial capital investment costs.

It should, however, be noted that the use of LEDFM alone should not be an alternative method but rather an adjuvant to the conventional $\mathrm{ZN}$ staining method, especially during the introductory phase. $\mathrm{ZN}$ method, even though archaic, is commonly used throughout the world and still remains a standard method against which new test methods should be compared. It is advisable that positive results by FM method should be confirmed by reexamination of the same smear by $\mathrm{ZN}$ technique when fluorescence method is being introduced. Apart from the fact that fluorescence fades with time, the FM method can give false positive results as compared to $\mathrm{ZN}$ staining. In a multi country cross-sectional evaluation of the two methods the researchers found out that even as FM had a higher sensitivity than $\mathrm{ZN}$, it also had a higher rate of false positives ${ }^{[11]}$.

\section{Conclusions}

LED- FM might not always increase sensitivity compared to $\mathrm{ZN}$. It is imperative that the introduction of LED-FM be accompanied by appropriate trainings and re-trainings and sustainable quality management, validation and monitoring of its field performance ${ }^{[11]}$. As a matter of fact, the institution and validation of an adequate, sustainable and frequent external quality assessment (EQA) system, including blinded rechecking, panel testing and on- site evaluation, should be seen as a pre- condition for scale up LED- FM ${ }^{[18]}$.

Table 1: Examination and grading of smears: NTP Guidelines.

\begin{tabular}{|c|c|c|}
\hline $\begin{array}{l}\text { IULTD/WHO Scale } \\
(1000 \times \text { field=HPF) } \\
\text { Result }\end{array}$ & $\begin{array}{l}\text { Bright field } \quad(1000 \mathrm{x}) \\
\text { magnification; } \\
\text { length=2cm=100HPF }\end{array}$ & $\begin{array}{l}\text { Fluorescence }(400 \mathrm{x}) \\
\text { magnification; } 1 \\
\text { lenght }=40 \\
\text { fields }=200 \mathrm{HPF}\end{array}$ \\
\hline Negative & Zero AFB/ 1 length & Zero AFB/1 length \\
\hline Scanty (actual count) & 1-9 AFB/100 fields & 1-19 AFB/ 1 length \\
\hline $1+$ & 10-99AFB/100 fields & 20-199AFB/1 length \\
\hline $2+$ & $\begin{array}{lll}1-10 & \text { AFB/field } & \text { on } \\
\text { average }\end{array}$ & $\begin{array}{l}5-50 \text { AFB/field on } \\
\text { average }\end{array}$ \\
\hline $3+$ & $\begin{array}{lll}\geq 10 & \mathrm{AFB} / \text { field } & \text { on } \\
\text { average } & & \end{array}$ & $\begin{array}{l}>50 \quad \text { AFB/field } \\
\text { average }\end{array}$ \\
\hline
\end{tabular}

Table 2: Results of smear Examinations by ZN and FM methods

\begin{tabular}{|l|l|l|}
\hline & ZN method & FM method \\
\hline Number of smears negative & $579(91.9 \%)$ & $575(91.3 \%)$ \\
\hline Number of smears positive & $51(8.1 \%)$ & $55(8.7 \%)$ \\
\hline Total number of smears & $630(100 \%)$ & $630(100 \%)$ \\
\hline
\end{tabular}




\section{References}

1. United States Agency for International Development (USAID) Nigeria (2012) HIV/AIDS and Tuberculosis.

2. WHO (2011) Sixteenth global report on Tuberculosis: Executive Summary.

3. United States Embassy Nigeria (2011) Nigeria HIV Fact Sheet, September 2011. http://nigeria.usembassy.org.

4. Federal Ministry of Health (FMOH)(2010) Department of Public Health: National Tuberculosis and Leprosy Control Programme (NTBLCP), Worker's Manual Revised $5^{\text {th }}$ Edition.

5. Steingart K R, Megan H, Virenne N G. Sputum processing method to improve the sensitivity of smear microscopy for tuberculosis : A Systematic Review. The Lancet Infect. Dis 2009: 6 (10): 664-6774.

6. Mark D P, Giorgio R, Alimuddin Z. Progress towards improved tuberculosis diagnostics for developing countries. Lancet 2006: 367: 942-43.

7. Allen J. A modified Ziehl - Neelse stain for mycobacteria. Medical Laboratory Sciences 1992: 49: 99-1

8. Infectious Disease Survey of America (IDSA). The Forum for Collaborative HIV Research. HIV/TB Co infection: Basic Facts, 2007.

9. International Union Against Tuberculosis and Lung Diseases (IUATLD) technical guide : sputum examination for tuberculosis by direct microscopy in low income countries. $5^{\text {th }}$ ed. 2000.

10. Cambanis A, Ramsay A, Wirkom V, Tata E, Cuevas L E. Investing time in microscopy : an opportunity to optimize smear based case detection of tuberculosis of tuberculosis. Int. J. Tuberc. Lung Dis 2007: 11: 40-45.

11. Cuevas L E, Vassin M A, Al-Sonboli, Lawson N, BAhader J. A multi-country non -inferioriy cluster randomize trial of front -loaded smear microscopy for the diagnosis of pulmonary tuberculosis. PLOS Med 2011: 8 (7): el000403.

12. Saroj H, Nital P, Bharti M, Sumit G, Vipin K, Leela V. Comparison of Ziehl- Neelsen and Auramine staining methods on direct and concentrated smears in clinical specimens. Indian Journal of Tuberculosis 2011: 58:72-76.

13. Reider H L. (1999) A Comparison of Fluorescence Microscopy with Ziehl Neelsen technique in the examination of Sputum for Acid Fast Bacilli. International Journal of Tuberculosis and Lung Disease 1999: 3 (12): 1101-1105.

14. Steingart K R, Ramsay A, Pai M. Optimizing sputum smear microscopy for the diagnosis of pulmonary tuberculosis. Expert Rev Anti Infect Ther 2007: 5 (3): 327-31.

15. Prasnthi K, Kumari A R. Efficacy of fluorochrome stain in the diagnosis of pulmonary tuberculosis patients coinfected with HIV. Indian J Med Microboil 2005: 23:179-85.

16. Ritu K, Anita P, Ashish K, Asthana, Agrawal R K. (2007). Comparison between $\mathrm{ZN}$ and Auramine $\mathrm{O}$ staining for detection of Acid Fast Bacilli. Asian Journal of Microbiology, Biotechnology and Environmental Sciences Paper 2007: 9 (4): 1035-1036.

17. Saroj G, Vivek H, Nirmala A R, Sangeetha K T, Asha S, Kamath B. A comparative study of auramine staining using LED fluorescent microscopy with $\mathrm{ZN}$ staining in the diagnosis of pulmonary tuberculosis. Journal of Evolution of Medical and Dental Sciences 2013: 2 (20): 3450-6.

18. Bonnet M, Gagnidzel L, Githui W, Guerin P J, Bonte L, Varaine F, Ramnsay A. Performance of LED-Based fluorescence Microscopy to diagnose tuberculosis in a peripheral health centre in Nairobi. PLOS One Journal 2011: 6(2): e17214. 